\title{
Artigo/Article
}

\section{Malária em mulheres de idade de 10 a 49 anos, segundo o SIVEP- Malária, Manaus, Amazonas, 2003-2006}

\author{
Malaria among women aged 10 to 49 years, according to SIVEP-Malaria, Manaus, State of \\ Amazonas, 2003-2006
}

\author{
Lesliane Balbino de Almeida ${ }^{1,2}$, Maria das Graças Vale Barbosa ${ }^{1,2,3}$ e Flor Ernestina Martinez-Espinosa ${ }^{2,3,4}$
}

\begin{abstract}
RESUMO
Introdução: O Sistema de Informação de Vigilância Epidemiológica SIVEP-Malária é um sistema de informação onde são notificados os casos de malária diagnosticados no Brasil, desde 2003. Este estudo analisou os casos de malária notificados em mulheres de 10 a 49 anos de idade, segundo a presença ou ausência de gestação no período de 2003-2006. Métodos: Foi solicitado à Fundação de Vigilância em Saúde, a autorização para avaliação dos dados. Resultados: Foram analisados 13.308 casos notificados no período, sendo 815 (6,1\%) registrados em gestantes. Houve diminuição gradativa do número absoluto de casos entre gestantes e não gestantes. Em relação à espécie, 14,3\% dos casos notificados foram causadas pelo Plasmodium falciparum; $85 \%$ pelo Plasmodium vivax e $0,6 \%$ pela associação dos dois. A frequência da infecção por Plasmodium falciparum foi maior entre gestantes que não gestantes $(\mathrm{p}>0,05)$. Embora a maior parte dos casos residam na zona leste da cidade, a zona oeste apareceu como o local de provável infecção em $39 \%$ dos casos. Observou-se picos endêmicos de malária entre as não gestantes nos meses de julho e agosto dos quatro anos analisados. Conclusões: Os dados mostram que o SIVEP-Malária é importante ferramenta para o conhecimento da distribuição da malária que deve ser usado para controle da endemia. Entretanto, os dados dos primeiros anos de funcionamento tiveram sua qualidade comprometida por falhas no preenchimento, usando o campo de notificação gravidez como exemplo.
\end{abstract}

Palavras-chaves: Malária. Gestante. Plasmodium sp. Fatores de riscos. Amazonas.

\begin{abstract}
Introduction: The SIVEP-Malaria Epidemiological Surveillance Information System has been in use for notification of malaria cases diagnosed in Brazil since 2003. This study analyzed malaria cases notified among women aged 10 to 49 years between 2003 and 2006, according to the presence or absence of pregnancy. Methods: Authorization to evaluate the data was requested from the Health Surveillance Foundation (FVS). Results: Over this period, 13,308 malaria cases were notified, of which $815(6.1 \%)$ were among pregnant women. There was a gradual decrease in the absolute numbers of cases among pregnant and non-pregnant women. Regarding species, $14.3 \%$ of the notified cases were caused by Plasmodium falciparum; $85 \%$ by Plasmodium vivax and $0.6 \%$ by both of them. The frequency of Plasmodium falciparum infection was greater among pregnant women than among non-pregnant women $(\mathrm{p}>0.05)$. Although most of the cases lived in the eastern zone of the city, the western zone appeared to be the likely location of infection in 39\% of the cases. Endemic peaks of malaria in July and August were observed among the non-pregnant women in all four years analyzed. Conclusions: The data showed that SIVEP-Malaria was an important tool for determining the distribution of malaria cases and that it should be used for controlling the endemic disease. However, the data from its first four years of operation showed that the quality was compromised by data entry failures, using the field of notification of pregnancy as an example.
\end{abstract}

Key-words: Malaria. Pregnant woman. Plasmodium sp. Risk factors. Amazonas.

1. Curso de Pós-Graduação em Medicina Tropical, Escola Superior de Ciências da Saúde, Universidade do Estado do Amazonas, Manaus, AM. 2. Gerência de Entomologia e Malária, Fundação de Medicina Tropical do Amazonas, Manaus, AM. 3. Centro de Pesquisas Leônidas e Maria Deane, Fundação Oswaldo Cruz, Manaus, AM. 4. Centro Universitário Nilton Lins, Manaus, AM.

Endereço para correspondência: Dra. Flor Ernestina Martinez-Espinosa. Av. Pedro Teixeira 25, Dom Pedro, 69040-000 Manaus, AM.

Tel: 5592 9902-1158

e-mail: florespinosa@gmail.com

Recebido para publicação em 01/07/2009

Aceito em 11/03/2010

\section{INTRODUÇÃO}

A malária constitui um grave problema de saúde pública, principalmente nas áreas endêmicas onde a doença é favorecida pelo clima tropical ${ }^{1}$, estudos ou dados sugerem associação entre a incidência da doença e a expansão ambiental urbana de maneira desordenada, sem planejamento e sob forma de invasões ${ }^{2}$.

A malária pode afetar o curso da gestação ocasionando aborto ou parto prematuro. Já a gestação torna a mulher mais vulnerável aos efeitos da doença fazendo com que as formas complicadas de malária sejam mais frequentes em gestantes ${ }^{3}$. Embora multifatorial, a anemia da gestante se vê agravada pela malária e tem um impacto significativo na saúde da mãe e do feto, sendo uma das suas complicações mais prevalentes, independente do cenário epidemiológico ${ }^{4,5}$. Nas gestantes com malária, anemia é comum em qualquer época da gestação, embora alguns estudos mostrem maior prevalência na primeira metade ${ }^{6}$ e outros estudos, realizados nas áreas de transmissão das Américas, mostrem maior frequência no último trimestre da gestação ${ }^{7-9}$.

As gestantes que vivem em áreas com menor risco de transmissão possuem baixa imunidade específica, ficando a mãe e o concepto alvos potenciais das formas complicadas de malária ${ }^{5,8,10}$. Portanto, é necessário realizar o diagnóstico precoce, instaurar um tratamento apropriado o mais rápido possível e fornecer um acompanhamento especial para as grávidas com malária, para evitar os efeitos adversos sobre a gestante e o concepto ${ }^{1}$. Atualmente, uma portaria do Ministério da Saúde obriga todos os postos de saúde responsáveis pelo controle pré-natal em áreas endêmicas de malária, a realizarem a gota espessa (método adotado oficialmente no Brasil para o diagnóstico da malária) em cada visita da gestante ao ambulatório ${ }^{11}$.

A Secretaria de Vigilância em Saúde implantou, no ano 2003, o Sistema de Informação de Vigilância Epidemiológica da Malária (SIVEP-Malária) na Região Amazônica, visando melhorar o fluxo, a 
qualidade e a oportunidade de informações entre os Municípios, Estado e União ${ }^{12}$. Este sistema foi criado aplicando-se conceitos modernos de tecnologia de informação, e desenvolvido em duas versões, local e on-line. A versão on-line (http//sis.saude.gov.br/ sivep-malaria) permite a digitação para aqueles que estão conectados na rede mundial de computadores e devidamente autorizados a operar o sistema, uma inovação tecnológica que permite agilidade na transferência de dados a partir de áreas de difícil acesso. $\mathrm{O}$ SIVEP-Malária é composto por módulos de notificação, módulos auxiliares para cadastrar localidades, laboratórios, unidades notificantes e agentes notificantes, além de dados para identificação do paciente, local provável onde ocorreu a infecção, resultado do exame laboratorial ${ }^{13-14}$.

O objetivo deste estudo foi utilizar as informações incluídas no SIVEP-Malária, no período de 2003-2006 para descrever a proporção de casos por idade, local de residência, local provável de infecção, frequência das espécies causadoras de malária, os principais aspectos da evolução temporal e da distribuição espacial da malária em mulheres de idade entre 10-49 anos, segundo a presença ou ausência de gestação.

\section{MÉTODOS}

Este é um estudo descritivo no qual foram incluídos 13.308 casos de malária em mulheres na faixa etária de 10 a 49 anos, registrados no SIVEP-Malária entre 2003 e 2006. Foi solicitado a Fundação de Vigilância em Saúde a autorização para avaliação dos casos. Os dados do SIVEP relativos à ocorrência de malária em mulheres pertencentes à faixa etária estudada foram obtidos, na forma de CD-ROM, diretamente junto à Coordenação Geral do Programa Nacional de Controle da Malaria (CGPNCM), Secretaria de Vigilância em Saúde/MS, prévio compromisso com o sigilo dos dados.

Foram descritas e analisadas as seguintes variáveis: presença de gestação, idade e data de diagnóstico, procedência e local provável da infecção. Para fins de distribuição geográfica, adotou-se a subdivisão da Cidade de Manaus em zonas (leste, oeste, norte e sul). Os casos de malária em gestantes e não-gestantes foram descritos através de suas frequências absolutas simples e relativas. Os parâmetros epidemiológicos foram descritos utilizando-se os programas Epi info 3.4.1, Tabwin 3.5 e Excel.

A média de idade das mulheres no momento da notificação do caso, a frequência de infecção por espécies e distribuição temporal e espacial dos casos, foram comparadas entre os grupos de gestantes e não-gestantes. Variáveis categóricas foram comparadas utilizando-se o teste do qui-quadrado de Pearson. Na análise de comparação das médias, utilizou-se o teste $t$ de Student. Valores de $p$ inferiores a 5\% foram considerados estatisticamente significativos. As variáveis com valores ignorados foram suprimidas da análise estatística.

\section{RESULTADOS}

Houve uma diminuição gradativa do número absoluto de casos de malária desde o primeiro até o último ano avaliado, mantendo-se a proporção de casos entre gestantes e não gestantes (Tabela $\mathbf{1}$ ).

Dos 13.308 casos de malária notificados nesse período, em mulheres de idade fértil, foram identificadas $815(6,1 \%)$ gestantes. As gestantes com malária apresentaram média de idade, no momento do
TABELA 1 - Distribuição anual de números de casos de malária em mulheres de 10-49 anos no período estudado, segundo dados do SIVEP-Malária.

\begin{tabular}{|c|c|c|c|c|c|c|}
\hline \multirow[b]{3}{*}{ Ano } & \multicolumn{6}{|c|}{ Gestantes } \\
\hline & \multicolumn{2}{|c|}{$\operatorname{sim}$} & \multicolumn{2}{|c|}{ não } & \multicolumn{2}{|c|}{ total } \\
\hline & $\mathrm{n}^{\mathrm{o}}$ & $\%$ & $\mathrm{n}^{\mathrm{o}}$ & $\%$ & $\mathrm{n}^{\mathrm{o}}$ & $\%$ \\
\hline 2003 & 183 & 4,3 & 4.034 & 95,7 & 4.217 & 100,0 \\
\hline 2004 & 239 & 7,0 & 3.161 & 93,0 & 3.400 & 100,0 \\
\hline 2005 & 226 & 6,9 & 3.044 & 93,1 & 3.270 & 100,0 \\
\hline 2006 & 167 & 6,9 & 2.254 & 93,1 & 2.421 & 100,0 \\
\hline Total & 815 & 6,1 & 12.493 & 93,9 & 13.308 & $\overline{100,0}$ \\
\hline
\end{tabular}

diagnóstico de (23,3 anos) quando comparada com as não-gestantes $(26,1$ anos $)(p<0,001)$. Na distribuição geral por faixa etária da ocorrência da doença, observou-se maior número de casos, na faixa etária entre 20 e 29 anos, $(\mathrm{p}<0,001)$, com mais de $50 \%$ dos casos de malária entre gestantes concentrados nessa faixa etária, sendo entretanto, mais uniforme nas diferentes faixas etárias entre as nãogestantes (Figura 1).

Em relação à espécie, 14,3\% dos casos notificados foram causado pelo Plasmodium falciparum; $85 \%$ pelo Plasmodium vivax e 0,6\% pela associação dos dois. A frequência da infecção por Plasmodium falciparum foi maior entre gestantes que não-gestantes $(\mathrm{p}>0,05)$ (Tabela 2).

De acordo com a zona de residência das pacientes, observou-se que existe uma distribuição desigual de casos, embora a maior parte dos casos residam na zona leste da cidade, a zona oeste aparece como o local mais provável de infecção em 39\% dos casos, seguida das zonas leste, norte e sul. Esta última conta com 196 residentes afetados, sugerindo um menor percentual de casos de malária em moradores desta região, entretanto, não houve associação estatisticamente significativa entre estar gestante e morar ou se infectar em um determinado local. No período avaliado, 446 pacientes ( 21 gestantes e 425 não gestantes) relataram a zona sul como local provável de infecção (Figura 2).

$\mathrm{Na}$ análise da série temporal, na subpopulação de gestantes, não se observa um padrão de concentração de casos ao longo dos anos avaliados. Em contraste, entre as não-gestantes, nota-se um aumento relativo sazonal e sistemático de casos nos quatro anos analisados, sempre ocorrendo entre os meses de julho e agosto (Figura 3).

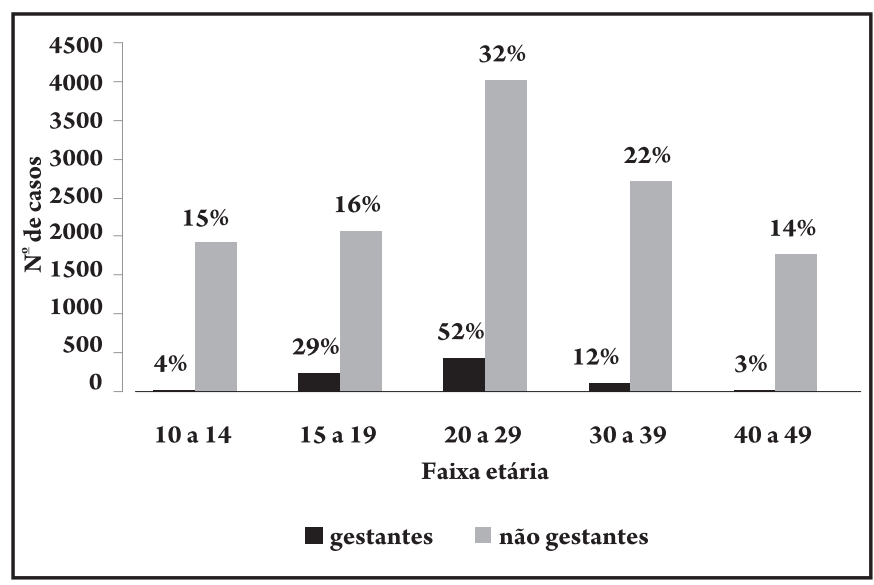

FIGURA 1- Distribuição de ocorrência de gestação por faixa etária nos casos notificadas com malária no município de Manaus-AM, 2003-2006. 
TABELA 2 - Ocorrência de infecção por Plasmodium falciparum, Plasmodium vivax e infecção mista em mulheres entre 10 a 49 anos do Município de Manaus, segundo presença ou ausência de gestação.

\begin{tabular}{|c|c|c|c|c|c|c|c|c|c|c|c|c|c|}
\hline \multirow[b]{3}{*}{ Ano } & \multicolumn{6}{|c|}{ Gestantes $\left(n^{\circ}=814\right)$} & \multicolumn{6}{|c|}{ Não-gestantes $(\mathrm{n}=12.494)$} & \multirow[b]{3}{*}{ p-valor } \\
\hline & \multicolumn{2}{|c|}{ Pf } & \multicolumn{2}{|c|}{$P v$} & \multicolumn{2}{|c|}{ mista } & \multicolumn{2}{|c|}{ Pf } & \multicolumn{2}{|c|}{$P v$} & \multicolumn{2}{|c|}{ mista } & \\
\hline & $\mathrm{n}^{\mathrm{o}}$ & $\%$ & $\mathrm{n}^{\mathrm{o}}$ & $\%$ & $\mathrm{n}^{\mathrm{o}}$ & $\%$ & $\mathrm{n}^{\mathrm{o}}$ & $\%$ & $\mathrm{n}^{\mathrm{o}}$ & $\%$ & $\mathrm{n}^{\mathrm{o}}$ & $\%$ & \\
\hline 2003 & 6 & 3,2 & 179 & 96,8 & 1 & 0,5 & 222 & 5,8 & 3.804 & 93,6 & 7 & 0,6 & $>0,2$ \\
\hline 2004 & 41 & 17,3 & 196 & 82,2 & 1 & 0,5 & 496 & 15,8 & 2.644 & 84,0 & 23 & 0,2 & $>0,05$ \\
\hline 2005 & 51 & 29,3 & 174 & 70,7 & 0 & 0,0 & 698 & 23,1 & 2.327 & 76,8 & 19 & 0,2 & $>0,09$ \\
\hline 2006 & 25 & 15,2 & 139 & 84,2 & 1 & 0,5 & 369 & 16,6 & 1.856 & 83,4 & 29 & 0,2 & $>0,09$ \\
\hline Total & 123 & 0,9 & 688 & 5,2 & 3 & 0,2 & 1.785 & 13,4 & 10.631 & 79,9 & 78 & 0,6 & $>0,05$ \\
\hline
\end{tabular}

Pf: Plasmodium falciparum, $P v$ : Plasmoduim vivax, mista: $P f+P v$.

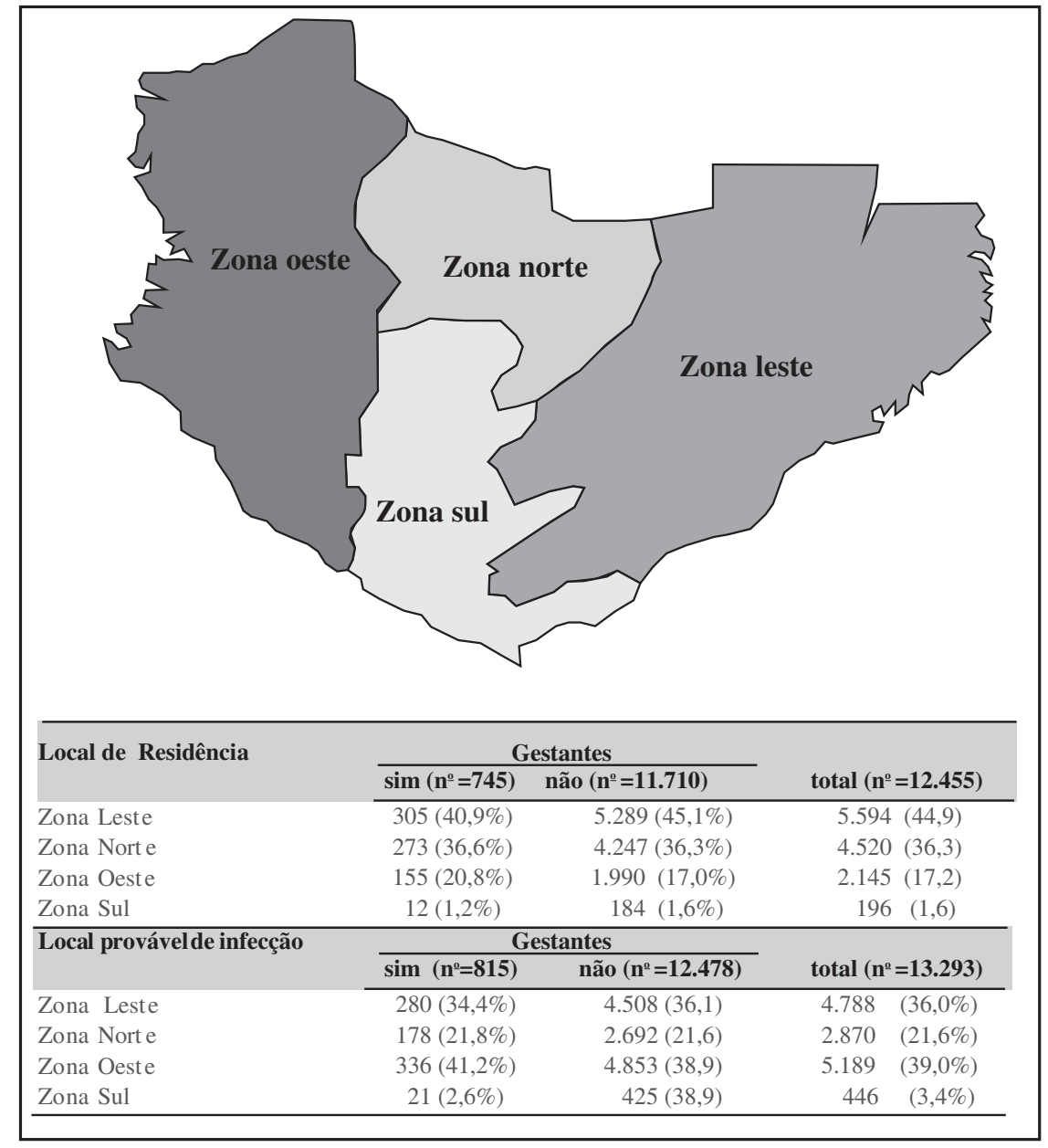

FIGURA 2 - Mapa de Manaus com a localização das zonas geográficas e distribuição das frequências de local de residência e local provável de infecção em mulheres gestantes e não-gestantes notificadas com malária entre 2003-2006.

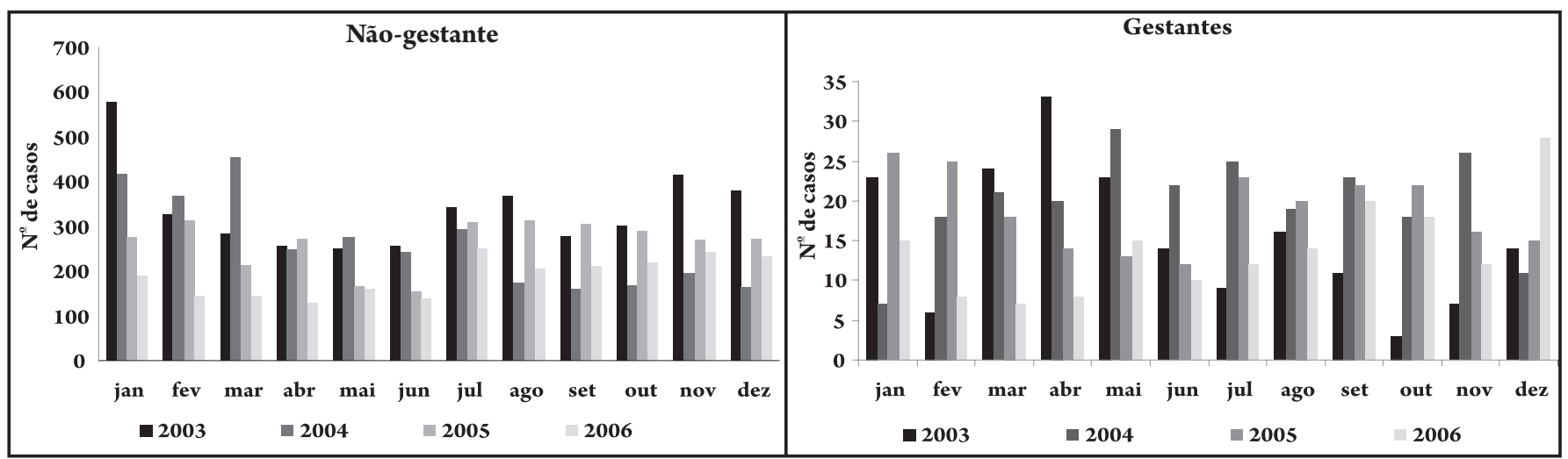

FIGURA 3 - Distribuiçao temporal mensal de casos de malaria em gestantes e não gestantes nos anos de 2003 a 2006 no município de Manaus. 


\section{DISCUSSÃO}

Nos países em desenvolvimento, como o Brasil, a notificação compulsória de doenças infecciosas é atividade essencial para permitir o planejamento de ações de controle das doenças que causam grande impacto sobre a saúde pública. A implementação destes sistemas abre a possibilidade, entre outras aplicações, da análise epidemiológica descritiva sistemática de um grande número de casos, com potencial capacidade de geração de hipóteses cuja investigação pode levar a melhoria nos sistemas de vigilância em saúde.

Este estudo descreve uma ampla amostra populacional de 13.308 casos de malária diagnosticados em Manaus, no período de 2003 a 2006, com ênfase na co-ocorrência de gestação. Nesta amostra populacional, foram identificados 815 (6,1\%) casos de malária em gestantes, uma proporção inesperadamente baixa, quando comparada com achados anteriores ${ }^{8,9}$. Isto pode indicar subnotificação do estado de gestação no momento do diagnóstico de malária, sugere a necessidade de intensificar o treinamento de quem coleta o dado, com a implantação de cursos periódicos de reciclagem, conscientização e consolidação dos procedimentos padrão.

Um aspecto a ser destacado neste estudo, é o número de dados, que permitiu análise comparativa de parâmetros epidemiológicos. Neste sentido, a comparação das médias de idade de diagnóstico entre gestantes e não gestantes afetadas por malária mostrou diferença estatisticamente significativa, com as gestantes adoecendo mais cedo do que as não gestantes ${ }^{15}$. A explicação mais provável para este achado é a gravidez que está associada à idade mais jovem entre as mulheres de idade fértil, outro sim, é possível que este efeito esteja associado ao nível educacional das subpopulações: populações com menor grau educacional estariam mais expostas à malária, ao mesmo tempo em que tenderiam a abrigar gestantes com idade mais precoce, conforme observado em estudo anterior realizado no município de Coari?

Atualmente, procura-se dar atenção especial à malária por Plasmodium falciparum, por ser esta a responsável pelo maior número de óbitos. Nossos dados não indicaram evidência estatisticamente significativa de que gestantes estariam mais suscetíveis à malária causada por Plasmodium falciparum, resultado que contrasta com estudos anteriores ${ }^{8,9}$. Esta discordância pode ser devido ao fato de que, no primeiro estudo a população foi recrutada na Fundação de Medicina Tropical do Amazonas ${ }^{8}$ que concentra casos de doença mais grave, portanto com maior probabilidade de serem causados por Plasmodium falciparum, mas o segundo estudo é de base populacional e não pode explicar-se com o mesmo argumento. É provável que o subregistro de gestação e a falta de procura ativa dos casos registrados no sivep podem ser responsáveis pela não associação entre gestação e infecção por Plasmodium falciparum. Outro achado interessante foi a queda na proporção de casos de Plasmodium falciparum no ano de 2006, após 3 anos consecutivos de aumento (2003-2005). Esta queda também foi observada na população geral e pode ser atribuída à implantação de novo esquema de tratamento para malária causada por Plasmodium falciparum em $2006^{16} \mathrm{com}$ Artemether $20 \mathrm{mg} /$ Lumefantrine $120 / \mathrm{mg}$ (Coartem ${ }^{\otimes}$ ), adicionalmente o diagnóstico precoce que é um dos alicerces do programa de malária permite que a infecção seja tratada antes que entrem na circulação as formas gametociticas responsáveis pela infecção no hospedeiro invertebrado impactando sobre a transmissão por esta espécie.

Na comparação do número de casos de acordo com local de residência, e local provável de infecção, a zona leste de Manaus surge como a que apresenta a maior proporção de moradoras com malária (gestantes e não-gestantes), e a zona oeste, como o local mais provável de infecção. Interessante notar que a zona com maior concentração de casos (zona leste) não é aquela com maior percentual de infecção (zona oeste).

Os 196 (1,6\%) casos registrados como local de residência a zona sul, não coincide com o histórico de endemicidade dessa área reconhecida como baixo risco ${ }^{17}$. Dessa forma, é importante destacar que, o relato de local provável de infecção na zona sul, dos 446 (3,4\%) casos, sugere que esses indivíduos possam ter se infectado em zonas de maior endemicidade - alto risco - uma vez que a zona sul é considerada área sem transmissão ${ }^{17}$. Pode-se especular que a ferramenta de coleta do dado local provável de infecção presente na ficha de notificação compulsória do SIVEP-Malária está sujeita a falhas de preenchimento que, por sua vez, podem induzir à incorreta interpretação da informação obtida.

Na distribuição temporal de casos por ano analisado para as duas subpopulações, observam-se frequentes oscilações na incidência da malária, seja em escala mensal ou anual. Picos de alta endemicidade podem ser distinguidos na subpopulação de não-gestantes no período entre julho e agosto, que coincide com o período final das chuvas, no qual a proliferação do vetor é maior ${ }^{18}$. A mesma observação não é possível para a subpopulação de gestantes, talvez devido à totalidade dos casos notificados.

O cumprimento das funções de Vigilância Epidemiológica depende da disponibilidade de informações que servem para subsidiar o desencadeamento das ações de planejamento em saúde. Contudo, as informações em saúde no Brasil necessitam de padronização e democratização. A abrangência e a qualidade dos sistemas de informações, administrados pelo Ministério da Saúde, vem aumentando ano a ano, permitindo a elaboração de trabalhos de alto valor acadêmicos e análises de extrema utilidade para o planejamento, organização e avaliação de serviços e programas por profissionais que constroem o dia-a-dia do Sistema Único de Saúde, nos diferentes municípios do país ${ }^{12,13,19}$.

Algumas limitações metodológicas deste estudo devem ser observadas; por exemplo, os dados foram obtidos a partir de fonte secundária, passível de modificação causada por preenchimento incorreto do SIVEP-Malária, hipótese reforçada pela frequente observação de campos ignorados, incompletos efalha no preenchimento. Além disso, fica a questão se o observado para o período entre 20032006 é representativo da situação atual da malária no município de Manaus. Apesar disso, os resultados aqui apresentados reforçam a noção de que a malária é um grave problema de saúde pública em Manaus, com destaque para a subpopulação de gestantes, sujeita a esquemas de tratamento limitados e potenciais complicações para gestante e concepto, que necessita de monitoramento constante em todas as zonas da cidade, inclusive, a zona sul, tradicionalmente, considerada de baixo risco ${ }^{17}$. É fundamental manter alerta os gestores municipais para a necessidade de priorizar políticas que envolvam ações preventivas, como saneamento, assistência básica de saúde e trabalho de ação social.

\section{AGRADECIMENTOS}

Agradecemos o Dr Marcelo Távora Mira, do Programa de PósGraduação em Ciências da Saúde da Pontifícia Universidade Católica do Paraná e Bolsista Visitante Sênior da FMTAM, pela contribuição da leitura crítica e preparo do manuscrito e ao Dr Rui Moreira Braz, Assessor Técnico do PNCM/MS/SVS, pela contribuição na aquisição dos dados sobre a malária. 


\section{CONFLITO DE INTERESSE}

Os autores declaram não haver nenhum tipo de conflito de interesse no desenvolvimento do estudo.

\section{REFERÊNCIAS}

1. Fundação Nacional de Saúde. Guia de Vigilância epidemiológica vol. II, Secretaria de Vigilância em Saúde, Ministério da Saúde, Brasília; 2003.

2. Alecrim WD, Gonçalves MJF. Non-planed Urbanization as a contributing for malaria incidence in Manaus - Amazonas, Brazil. Rev Saúde Publica 2004; 6:156166

3. Martinez-Espinosa FE, Daniel-Ribeiro CT, Alecrim WD. Malaria during pregnancy in a reference centre from the Brazilian Amazon: unexpected increase of Plasmodium falciparum infections. Mem Inst Oswaldo Cruz 2004; 99:19-21.

4. Brabin BJ. An analysis of malaria in pregnancy in Africa. Bull World Health Organ 1983; 61:1005-1016.

5. Menendez C. Malária During Pregnancy: A Priority area of malaria research and control. Parasitol Today 1995; 11:178-183.

6. Nosten F, Kullie F, Maelankirri L, Decludt B, White NJ. Malaria during pregnancy in an area of unstable endemicity. Trans R Soc Trop Med Hyg 1991; 85:424429

7. Jarude R, Trindade R, Tavares-Neto J. Malária em grávidas de uma maternidade pública de Rio Branco (Acre, Brasil). Rev Bras Ginecol Obstet 2003; 25:149154

8. Martinez-Espinosa FE. Malária na gravidez: Estudo de Pacientes do Instituto de Medicina Tropical do Amazonas, Brasil, 1990-1997 [dissertação]. [Rio de Janeiro (RJ)]: Fundação Oswaldo Cruz; 1998.

9. Martinez-Espinosa FE. Malária e Gravidez na Região Amazônica: Prevalência de Infecção em mulheres de idade fértil de Coari 2001-2002 [tese]. [Rio de Janeiro (RJ)]: Fundação Osvaldo Cruz; 2003.

10. Ávila SLM. Malária. In: Ferreira AW, editor. Diagnóstico laboratorial das principais doenças infecciosas e auto-imunes. Editora Guanabara Koogan, Rio de Janeiro; 1996. p.157-158.

11. Secretaria de Vigilância em Saúde. Importância da gota espessa nas consultas de pré-natal. Programa Nacional de Controle da Malária Coordenador Geral, Ministério da Saúde, Nota Técnica - CGPNCM/DIGES/SVS/MS; 2006.

12. Braz RM, Andreozzi VL, Kale PL. Detecção precoce de epidemias de malária no Brasil: uma proposta de automação. Epidemiol Serv Saúde 2006; 15:21-33.

13. Secretaria Executiva. Política Nacional de Informação e Informática em Saúde. Departamento de Informação e Informática do SUS, Ministério da Saúde, Brasília; 2004

14. SIVEP-Malária. Notificação de casos. Relatório. Brasília: Secretária de Vigilância em Saúde/Sistema de Informações de Vigilância em Saúde/Ministério de Saúde; 2006.

15. Chagas ECS, Nascimento CT, Santana FS, Bôtto-Menezes CH, MartinezEspinosa FE. Malária durante a gravidez: efeito sobre o curso da gestação na região amazônica. Rev Panam Salud Publica, Washington. 2009; 26:203208.

16. Medeiros MB, Alecrim MGC, Alecrim WD, Santana FS. Dados preliminares do estudo comparativo da eficácia de Quinino-Doxiciclina e ArtemeterLumefantrina $\left(\right.$ Coartem $\left.^{\circledast}\right)$ para o Tratamento de Malária por Plasmodium falciparum não Complicada no Município de Coari, AM. In: XLlI Congresso Soc Bras Med Trop, Teresina-PI; 2006.

17. Saraiva MGG, Amorim RDS, Moura MAS, Martinez-Espinosa FE, Barbosa MGV Expansão urbana e distribuição espacial da malária no município de Manaus, Estado do Amazonas. Rev Soc Bras Med Trop 2009; 42:515-522.

18. Klein TA, Lima JBP. Seasonal distribution and biting patterns of anopheles mosquitoes in Costa Marques, Rondônia, Brasil. J Am Mosq Control Assoc 1990; 6:700-707.

19. Tauil P L.A importância do uso de sistemas de informação em estudos e pesquisas na área da epidemiologia. Epidemiol Serv Saúde, Brasília, DF. 2004; 13:5-6. 